\title{
Orbitofrontal dysfunction predicts poor prognosis in chronic migraine with medication overuse
}

\author{
Marian Gómez-Beldarrain • María Carrasco • \\ Amaia Bilbao · Juan C. García-Moncó
}

Received: 3 March 2011/Accepted: 31 March 2011/Published online: 17 April 2011

(c) The Author(s) 2011. This article is published with open access at Springerlink.com

\begin{abstract}
Chronic migraine patients are at risk of developing a medication overuse. Brain functional studies in these patients have demonstrated an orbitofrontal hypometabolism, persistent after overuse cessation. Orbitofrontal dysfunction is also present in addiction and thus could predispose migraineurs to medication overuse. The aim of this study was to investigate if orbitofrontal dysfunction can be demonstrated in patients with chronic migraine and medication overuse by performing a systematic neuropsychological evaluation focused on tests that assess frontal lobe function. Second, to establish whether it is related to the outcome of these patients. We prospectively studied 42 chronic migraine patients with medication overuse, 42 episodic migraineurs and 41 controls on a battery of neuropsychological tasks evaluating the orbitofrontal and dorsolateral functioning. Depression, anxiety, and personality traits were also assessed. Chronic migraineurs with medication overuse showed a significant impairment in orbitofrontal task performance and higher depression scores as compared to episodic migraineurs and controls. Dorsolateral dysfunction was present in both groups of migraneurs, who also had higher rates of anxiety as compared to controls. After 1 year of follow-up, migraine patient's outcome was classified according to
\end{abstract}

Electronic supplementary material The online version of this article (doi:10.1007/s10194-011-0340-6) contains supplementary material, which is available to authorized users.

M. Gómez-Beldarrain ( $₫)$ · M. Carrasco · J. C. García-Moncó Service of Neurology, Hospital de Galdacano, 48960 Galdacano, Vizcaya, Spain

e-mail: mgomezab@sarenet.es

A. Bilbao

Fundación Vasca de Innovación e Investigación Sanitarias (BIOEF), 48150 Sondika, Vizcaya, Spain their medication overuse status. A negative outcome that included persistent or new-onset medication overuse was present in $34 \%$ of migraineurs and was associated with baseline poor orbitofrontal task performance, and with mild dorsolateral dysfunction, higher rates of depression, anxiety and neuroticism-anxiety traits. Formal education and years with migraine did not influence outcome. Orbitofrontal dysfunction is present in patients with chronic migraine and medication overuse, and associates with a poor outcome at 1 year of follow-up. Neuropsychological evaluation in migraine may help to detect patients prone to overuse so that appropriate therapeutic attitudes can be taken.

Keywords Chronic migraine - Medication overuse · Episodic migraine $\cdot$ Orbitofrontal cortex .

Dorsolateral cortex

\section{Introduction}

Medication overuse occurs predominantly in patients with a primary headache disorder, particularly migraine patients with a high attack rate [1-4]. Approximately, $10 \%$ of migraine patients develop chronic migraine $(\mathrm{CM})$, a condition characterized by an insidious increase of headache frequency and intensity that often results in medication overuse and deterioration of the patient' quality of life, also representing a significant economic burden [5].

The pathophysiology of this challenging condition is unclear, but several lines of evidence suggest that neuropsychological mechanisms, particularly orbitofrontal (OF) cortex dysfunction, are involved. A PET study that used 18-FDG in 16 chronic migraineurs with medication overuse revealed a persistent $\mathrm{OF}$ hypofunction before and 
3 weeks after medication withdrawal as compared to healthy controls [6].

OF hypofunction impairs reward and decision-making mechanisms [7, 8], occurs in substance abusers, and could contribute to medication overuse in CM patients. Dorsolateral (DL) executive dysfunctions, including planning, problem solving, and set-shifting tasks have also been described in patients with migraine $[9,10]$, although not clearly related to medication overuse.

Taken together, these findings suggest that medication overuse might be a consequence of a susceptibility to substance abuse due to prefrontal, particularly OF, dysfunction [6].

In this study, we sought to investigate if OF dysfunction can be demonstrated by performing a systematic neuropsychological evaluation focused on tests that assess prefrontal lobe functions. We also tried to determine if there is a relationship between OF functioning and the outcome of migraine patients in terms of medication overuse.

To this end, we compared a group of chronic migraineurs with medication overuse with episodic migraineurs and with controls, and followed them for 1 year.

\section{Design and methods}

\section{Subjects}

Three groups of individuals were included in this study:

(a) Forty-two patients suffering from chronic migraine and medication overuse (chronic migraine group$\mathrm{CM}$ group), were consecutively recruited from the Neurology department at our institution. Inclusion criteria were age 55 or younger suffering from chronic migraine and medication overuse according to the current criteria of the International Headache Society [3, 11], i.e., definite or probable migraine headaches present on $>15$ days/month for $>3$ months and with an intake of simple analgesics on $\geq 15$ days per month or of any combination of ergotamine, triptans, analgesics, and/or opioids on $\geq 10$ days per month on a regular basis for $>3$ months. Exclusion criteria included a past history of any neurologic disease different from migraine or a past history of any psychiatric disorder, except for depression or anxiety. We also excluded patients with other chronic pain conditions or under medication for general medical diseases or taking psychotropic drugs.

(b) Forty-two consecutive patients with episodic migraine without aura [11] (episodic migraine group-EM group).The same exclusion criteria as for the CM group were applied. (c) Forty-one healthy subjects (control group-CG group), specifically without headache disorder or any other neurological or psychiatric disease, and under no psychoactive therapy.

All participants had a normal neurologic examination. All migraineurs had a normal brain MRI or CT scan. Only six chronic migraine patients and four patients with episodic migraine had small white matter hyperintensities consistent with those described in patients with migraine. After enrolment, they kept a headache calendar to register the number of days with headache and to keep a medication record.

Instructions on how to manage a migraine attack with NSAIDs and triptans were given to all patients, and appropriate preventive medication was selected or modified as needed on an individual basis. We avoided using opioids in these patients. Patients in the CM group were advised to cease medication overuse and were managed in an outpatient setting.

Patients (CM and EM) were followed for 1 year with scheduled visits at 3-month intervals. Special emphasis was placed in evaluating the status with regards to medication overuse (i.e., whether overusers converted to a non-overuser state and vice versa).

At the end of the follow-up, we classified migraine patients in two groups in terms of medication overuse: those with a positive outcome (EM patients that remained episodic and without medication overuse, and CM overusing medication that ceased overuse) and those with a negative outcome (overusers that persisted on this status after 1 year of follow-up, and EM patients that started overusing). We analyzed the differences in the baseline task performance between both groups and whether the outcome was related with baseline prefrontal functioning.

The study was approved by the Ethics Committee of our Institution and all participants signed a written consent according to the Helsinki recommendations.

Neuropsychological evaluation

During examination, headache was assessed by a visual analogue scale ranging from zero (no pain) to ten (worst headache); patients scoring $\geq 4$ were asked to perform the evaluation at some other time. The examination was carried out in two sessions of $1 \mathrm{~h}$ duration.

\section{Orbitofrontal assessment}

The three following tasks, that have been shown to be sensitive to ventromedial and OF damage, were employed [12-17]. 
1. "Reading the Mind in the Eyes" This task consists of photographs of the eye region of 36 different faces. The subject is required to make a choice between four words printed on top and bottom of the picture, and choose the one that best describes the feeling or thought of the subject in the photograph.

2. "Faux-Pas" In this test, the subject is read ten stories that contain a social Faux-Pas and ten stories describing a minor conflict, but in which no Faux-Pas is committed. The text of each story is placed in front of the subject so as to reduce the demands on working memory. The subject should identify the Faux-Pas stories. If a Faux-Pas is identified the subject is asked three questions: "Why shouldn't they have said what they did?"; "Why do you think they did it?"; and "How do you think the person affected by the FauxPas feel?" In every story memory questions (control questions) are asked to ensure the subject comprehension (See supplementary material).

3. The "Empathy Quotient" Empathy allows us to understand the intentions and feelings of others and predict their behaviour. We employed this self-report questionnaire for use in adults with normal intelligence. It contains 40 empathy items and 20 fill/control items. On each empathy item, a score 2, 1 or 0 can be obtained, so the maximum score of the empathy quotient is 80 .

\section{Dorsolateral assessment}

It was evaluated by standard tasks designed to measure executive function that depends on the dorsolateral frontal region.

1. Wisconsin card sorting test (WCST) (Heaton 1981, Psychological Assessment Resources). We employed a computerized version of this classic task measuring the ability to display flexibility in the face of changing schedules of reinforcement.

2. Letters and Numbers (WAIS-III 1997, The Psychological Corporation): This is a working memory test measuring the ability to manipulate a progressively longer piece of information.

3. Trail-making test (TMT) B: The TMT provides information on visual search, scanning, speed of processing, mental flexibility, and executive functions [18].

\section{Personality}

Zuckermann-Kuhlman III [19]: Self-administered 99-item questionnaire measuring impulsive sensation seeking, neuroticism-anxiety, aggression-hostility, activity, sociability and infrequency (these items detect subjects who responded carelessly).

\section{Emotional state}

Beck depression and anxiety inventories [20]. These questionnaires consist of 21 , self-administered items about how the subject has been feeling in the last week. Each question has a set of at least four possible choices ranging in intensity.

\section{Statistical analysis}

Descriptive statistics included frequencies and percentages, means, standard deviations (SD), and medians and range. Demographic characteristics and migraine information were compared between patients with chronic migraine and medication overuse, patients with episodic migraine and controls. Neuropsychological tests, and depression, anxiety and personality tests were also compared between the three groups. To compare categorical variables Chi-square and Fisher's exact tests were used, and for continuous variables the analysis of variance or the non-parametric KruskalWallis test was performed. The general lineal model was used to compare the neuropsychological tests between the three groups adjusting by age and depression, when necessary. Finally, all the neuropsychological tests were compared between the patients with positive course of their clinical status at 1 year of follow-up and those with negative course. For this comparison, the Student $t$ test or the Wilcoxon test were performed. Kolmogorov-Smirnov test was employed to establish the normality of the distribution of the variables.

Effects were considered significant at a $p$ value $<0.05$. All statistical analyses were performed using SAS for Windows statistical software, version 9.1 (SAS Institute, Inc., Carey, NC, USA).

\section{Results}

The main characteristics of the three groups are depicted on Table 1.

As expected, there was a female predominance in all groups $\left(\chi_{(2)}^{2}=5.75, p=0.0563\right)$, and chronic migraine patients with medication overuse were older than episodic migraine patients and controls $\left(\chi_{(2)}^{2}=6.70, p=0.0350\right)$. There were no significant differences in education years $\left(\chi_{(4)}^{2}=4.52, p=0.3397\right)$ or in years with headache $\left(\chi_{(2)}^{2}=2.14, p=0.3425\right)$ between CM and EM.

CM patients abused from NSAIDs, triptans, simple analgesics and a variable combination of the above. As 
Table 1 Demographics and migraine information for patients and controls

\begin{tabular}{|c|c|c|c|}
\hline & Chronic migraine with drug overuse & Episodic migraine & Controls \\
\hline$N$ (females) & $42(39)$ & $42(35)$ & $41(30)$ \\
\hline Age, mean (SD), years & $41.21(8.20)$ & $36.19(8.66)$ & $37.12(8.59)$ \\
\hline \multirow[t]{3}{*}{ Years of education } & $<12$ years $(17)$ & $<12$ years $(16)$ & $<12$ years $(9)$ \\
\hline & $12-16$ years $(16)$ & $12-16$ years $(14)$ & $12-16$ years $(17)$ \\
\hline & $>16$ years $(9)$ & $>16$ years $(12)$ & $>16$ years $(15)$ \\
\hline \multirow[t]{3}{*}{ Years with migraine } & $<5$ years $(5)$ & $<5$ years $(9)$ & - \\
\hline & $5-15$ years $(13)$ & $5-15$ years $(15)$ & \\
\hline & $>15$ years $(24)$ & $>15$ years $(18)$ & \\
\hline Medication overuse & $\begin{array}{l}\text { NSAIDs (21), triptans (3), simple analgesics (4), } \\
\text { combination of the above (14) }\end{array}$ & - & - \\
\hline Preventive medication & $\begin{array}{l}\text { Combination }^{\mathrm{a}}(15) \text {, amitriptyline (4), } \\
\text { topiramate (4), zonisamide (1), beta-blockers (3), } \\
\text { lamotrigine (1), flunaricine (1), } \\
\text { SSRI (2), no treatment (11) }\end{array}$ & $\begin{array}{l}\text { No treatment (26), beta-blockers (7), } \\
\text { amitriptyline (7), flunaricine (2) }\end{array}$ & - \\
\hline \multirow[t]{3}{*}{ Follow-up at 1 year } & Overuse persisted (24) & Continued without overuse (35) & - \\
\hline & Ceased overuse (16) & Started overuse (3) & \\
\hline & Lost to follow-up (2) & Lost to follow-up (4) & \\
\hline
\end{tabular}

NSAIDs Non-steroidal anti-inflammatory drugs, SSRI selective serotonin reuptake inhibitors

${ }^{a}$ More than one drug (valproic acid, zonisamide, topiramate, beta-blockers)

mentioned before, we avoid using opioids in these patients, the reason why these drugs are not represented here.

Different preventive medications were or had been used in the two groups of migraineurs. Twenty-six EM patients were not on preventive medication due to the infrequent headaches, and $11 \mathrm{CM}$ patients refused treatment due to the lack of consistent efficacy of prior preventive therapies.

Neuropsychological examination (Table 2)

In terms of OF evaluation, the Faux-Pas test revealed significant differences, with the CM group performing worse than the EM group and controls. CM patients detected less Faux-Pas situations $\left(\chi_{(2)}^{2}=6.71, p=0.0349\right)$ and detected a non-existent intentionality to a greater extent than the other two groups $\left(\chi_{(2)}^{2}=11.39, p=0.0034\right)$. Other OF tests did not disclose significant differences. The adjusted models showed that depression was significantly associated with the task Reading the Mind in the Eyes, and in spite of this adjustment, there were still no significant differences among the three groups.

DL examination found that both groups of migraine patients performed worse than controls in the Letters and Numbers test $\left(F_{(2,121)}=5.62, p=0.0046\right)$. CM patients had also significantly more difficulty in reaching the first category on the WCST $\left(\chi_{(2)}^{2}=9.82, \mathrm{p}=0.0074\right)$. The adjusted models showed that age was significantly associated with all the tasks, and depression only with Letters and Numbers and categories acquired on the WCST. After the corresponding adjustments, the results kept equal except for Trail Making B. We found that CM group performed significantly worse than controls $(p=0.0236)$.

Emotional and personality measures (Table 3)

Depression scores of CM patients were significantly higher than the other two groups $\left(\chi_{(2)}^{2}=17.65, p=0.0001\right)$, whereas both CM and EM showed higher anxiety scores than controls $\left(\chi_{(2)}^{2}=10.90, \mathrm{p}=0.0043\right)$. No significant differences were found on personality traits.

Follow-up (Table 4)

After 1 year of follow-up, six patients were lost, two in the CM group and four in the EM group (they refused to attend the appointments). Twenty-four of the CM patients were still abusing $(60 \%)$ and 16 had stopped medication overuse. In the EM group, three patients had started medication overuse. Overall, 27 migraine patients (34\%) showed a negative outcome, and 51 had a positive one (see definitions in methods).

We compared the baseline tasks performed by patients with negative and positive outcome. Patients with a positive outcome had performed significantly better on the three tasks measuring OF functioning with significant differences being present in all subtests of the Faux-Pas, the "Reading the mind in the eye" test and the Empathy Quotient.

No differences were found in tasks measuring DL function, except for one subtest of the WCST, where patients with a positive outcome performed better than 
Table 2 Neuropsychological tests results
Chronic migraine with Episodic migraine Controls $p$

drug overuse $(n=42) \quad(n=42)$

\begin{tabular}{|c|c|c|c|c|c|}
\hline \multirow{5}{*}{$\begin{array}{l}\text { Values expressed as median } \\
\text { (range) }\end{array}$} & & \multicolumn{2}{|l|}{ arug overuse $(n-42)$} & \multicolumn{2}{|l|}{$(n=41)$} \\
\hline & \multicolumn{5}{|l|}{ Orbitofrontal tasks } \\
\hline & \multicolumn{5}{|l|}{ Faux-Pas } \\
\hline & Faux-Pas Composite Score & $40(0-59)$ & $47(0-60)$ & $42(25-59)$ & 0.0397 \\
\hline & Detected Faux-Pas & $8(0-10)$ & $9(0-10)$ & $8(5-10)$ & 0.0349 \\
\hline \multirow{2}{*}{$\begin{array}{l}\text { WCST Wisconsin card sorting } \\
\text { test }\end{array}$} & Control questions & $40(38-40)$ & $39.5(37-40)$ & $40(37-40)$ & 0.2115 \\
\hline & Intentionality $^{\mathrm{a}}$ & $6(0-10)$ & $7(0-10)$ & $7(3-10)$ & 0.0034 \\
\hline \multirow{7}{*}{$\begin{array}{l}\text { a Subjects answered the } \\
\text { question: Why do you think } \\
\text { someone did the Faux-Pas? The } \\
\text { patient gives an incorrect } \\
\text { answer when he/she detects } \\
\text { intention in the Faux-Pas (which } \\
\text { was designed as unintentional); } \\
\text { the lower the score, the worse } \\
\text { performance }\end{array}$} & Empathy $^{\mathrm{b}}$ & $7(0-10)$ & $8(0-10)$ & $8(4-10)$ & 0.0825 \\
\hline & Reading the Mind in the Eyes & $25(18-31)$ & $26(14-32)$ & $27(16-35)$ & 0.2035 \\
\hline & Empathy quotient & $47(20-65)$ & $42(28-59)$ & $47.5(21-70)$ & 0.2970 \\
\hline & Dorsolateral tasks & & & & \\
\hline & Trail Making B & $74(37-210)$ & $68(30-203)$ & $64(30-119)$ & 0.1306 \\
\hline & Letters and Numbers & $10(4-19)$ & $10.5(4-20)$ & $13(6-21)$ & 0.0046 \\
\hline & WCST & & & & \\
\hline \multirow{3}{*}{$\begin{array}{l}\text { Subjects answered the } \\
\text { question: How do you think the } \\
\text { person affected by the Faux-Pas } \\
\text { feels? }\end{array}$} & Categories acquired & $3(0-5)$ & $4(1-5)$ & $4(0-6)$ & 0.1020 \\
\hline & Perseverative errors & $7(2-21)$ & $6(3-29)$ & $5(0-22)$ & 0.1116 \\
\hline & First category & $12(10-65)$ & $11(10-33)$ & $12(10-65)$ & 0.0074 \\
\hline
\end{tabular}

Table 3 Depression, anxiety, and personality test results

Values expressed as median (range)

\begin{tabular}{lllll}
\hline & $\begin{array}{l}\text { Chronic migraine with } \\
\text { drug overuse }(n=42)\end{array}$ & $\begin{array}{l}\text { Episodic migraine } \\
(n=42)\end{array}$ & $\begin{array}{l}\text { Control } \\
(n=41)\end{array}$ & $p$ \\
\hline Beck Depression Inventory & $13(0-44)$ & $6(0-27)$ & $5(0-22)$ & 0.0001 \\
Beck Anxiety Inventory & $12(1-51)$ & $11(0-43)$ & $6(0-35)$ & 0.0043 \\
Zuckerman-Kuhlman personality questionnaire & & & \\
Impulsive sensation seeking & $4(0-15)$ & $3(0-11)$ & $2.5(0-11)$ & 0.4670 \\
Neuroticism-anxiety & $8(1-19)$ & $9(0-16)$ & $5.5(1-16)$ & 0.0568 \\
Aggression-hostility & $7(0-14)$ & $7(2-13)$ & $7(2-12)$ & 0.6622 \\
Activity & $6(1-16)$ & $7(1-15)$ & $6.5(1-14)$ & 0.2660 \\
Sociability & $6(0-9)$ & $6(0-14)$ & $6(0-12)$ & 0.1825 \\
Infrequency & $1(0-8)$ & $1(0-7)$ & $2(0-6)$ & 0.3770 \\
\hline
\end{tabular}

those with a negative one in reaching the first category ( $U=1268, p=0.0112)$.

Patients with a negative evolution were significantly more depressed ( $U=1207, p=0.0010)$ and more anxious ( $U=1143, p=0.0053$ ) than the positive outcome group.

Regarding personality traits, patients with a negative course scored significantly higher than those with a positive course in the neuroticism-anxiety factor $\left(t_{(67)}=2.11\right.$, $p=0.0384)$.

There were no between-group differences groups in terms of age, years of migraine or years of formal education.

\section{Discussion}

Our results confirm the presence of OF dysfunction in $\mathrm{CM}$ patients with medication overuse from a neuropsychological perspective, and suggest that OF dysfunction heralds a poor prognosis in terms of medication overuse in these patients. In agreement with some clinical [9] and radiological studies [21], we also found a mild DL dysfunction in migraineurs (independent of overuse) as compared to controls. Migraine patients did also show greater anxiety scores than controls, and overusers were more depressed than the other two groups. The comorbidity of migraine with depression has led to suggest a shared pathogenesis [22].

In keeping with other studies, no differences were found in personality traits between migraine patients and controls [23]. We did not observe a relationship between OF dysfunction and the patients' education level or years of migraine, which in some studies have been suggested as risk factors for poor prognosis [24]. Some authors have proposed that rather than migraine duration it is the frequency and severity of the attacks what determines a malfunctioning of the circuitries [25] implicated in pain 
Table 4 Outcome after 1 year of follow-up
Values expressed as median (range)

${ }^{\text {a }}$ Patients with chronic headache that stopped medication overuse and patients with episodic headache who persisted without overuse

${ }^{b}$ Patients with chronic headache that persisted overusing medication and patients with episodic headache who converted into chronic migraine with medication overuse

\begin{tabular}{|c|c|c|c|}
\hline & $\begin{array}{l}\text { Positive outcome } \\
(n=51)^{\mathrm{a}}\end{array}$ & $\begin{array}{l}\text { Negative outcome } \\
(n=27)^{\mathrm{b}}\end{array}$ & $p$ \\
\hline \multicolumn{4}{|l|}{ Orbitofrontal tasks } \\
\hline \multicolumn{4}{|l|}{ Faux-Pas (FP) } \\
\hline FP Composite Score & $46(0-60)$ & $39(0-52)$ & 0.0054 \\
\hline Detected FP & $9(0-10)$ & $8(0-10)$ & 0.0176 \\
\hline Who committed the FP & $8(0-10)$ & $7.5(0-10)$ & 0.0329 \\
\hline Why is it a FP & $8(0-10)$ & $7(0-10)$ & 0.0336 \\
\hline Why someone did the FP & $6(0-10)$ & $4(0-8)$ & 0.0087 \\
\hline Intentionality & $7(0-10)$ & $5(0-9)$ & 0.0134 \\
\hline Empathy & $8(0-10)$ & $7(0-10)$ & 0.0150 \\
\hline Reading the Mind in the Eyes & $27(17-32)$ & $23(14-31)$ & 0.0018 \\
\hline Empathy Quotient & $45(31-65)$ & $44.5(20-60)$ & 0.0368 \\
\hline \multicolumn{4}{|l|}{ Dorsolateral tasks } \\
\hline Trail Making B & $68(30-203)$ & $79.5(39-210)$ & 0.0803 \\
\hline Letters and Numbers & $10(4-20)$ & $9.5(4-19)$ & 0.4009 \\
\hline \multicolumn{4}{|l|}{ WCST } \\
\hline Categories acquired & $4(0-5)$ & $3(0-5)$ & 0.0584 \\
\hline Perseverative errors & $6(3-29)$ & $7(2-20)$ & 0.4625 \\
\hline First category & $11(10-65)$ & $12(10-65)$ & 0.0112 \\
\hline Beck Depression Inventory & $8.5(0-27)$ & $15.5(1-44)$ & 0.0010 \\
\hline Beck Anxiety Inventory & $10(0-43)$ & $17.5(2-51)$ & 0.0053 \\
\hline \multicolumn{4}{|c|}{ Zuckerman-Kuhlman personality questionnaire } \\
\hline Impulsive sensation seeking & $3(0-11)$ & $4.5(0-15)$ & 0.8960 \\
\hline Neuroticism-anxiety & $8(0-16)$ & $11.5(1-19)$ & 0.0384 \\
\hline Aggression-hostility & $6(0-13)$ & $7.5(3-14)$ & 0.2075 \\
\hline Activity & $7(1-16)$ & $6(1-14)$ & 0.8264 \\
\hline Sociability & $6(0-14)$ & $4.5(0-9)$ & 0.0805 \\
\hline Infrequency & $1(0-8)$ & $1(0-6)$ & 0.4290 \\
\hline
\end{tabular}

medication overuse, and $60 \%$ of the $\mathrm{CM}$ patients persisted overusing medication [27]; it should be noted, however, that medication overuse fluctuated throughout the year in some patients. We observed that those patients with a negative outcome performed significantly worse at baseline than those with good outcome on the three OF tasks, i.e., patients with poor initial OF tests performance had a significantly higher risk of medication overuse, even if they were not abusing at study onset.

Our results and other studies [6] suggest that OF malfunctioning is present in the $\mathrm{CM}$ patients with medication overuse and predicts a negative outcome. Whether this $\mathrm{OF}$ dysfunction is the cause or is secondary to the abuse is an unsolved question but we suggest that this dysfunction is prior to abuse, since those EM patients with the worst baseline OF tests scores developed medication overuse.

The reason for this dysfunction is unclear. It could be speculated that as a result of a particular genetic background and gender (female), environmental factors (low socioeconomic status and education level, obesity), and headache characteristics (frequency, intensity and duration of pain) 
there would be a poorly developed prefrontal circuitry that predisposes these patients to medication overuse. Some genetic factors for medication overuse have also been described. Specifically, a polymorfism in the brain-derived neurotrophic factor (BDNF) gene (Val66Met) has been found as a significant independent predictor of analgesic consumption in patients with CM [28]. BDNF is involved in central pain sensitization and long-term potentiation. Also, wolframin His611 Arg polymorphism influences medication overuse headache. Patients with chronic migraine and medication overuse harbouring that polymorphism showed a higher monthly medication consumption that non carriers. Wolframin (WFS1) is involved certain psychiatric illness and dependence behaviour [29]. Other polymorphisms in dopamine or serotonine receptors might be involved as these neurotransmitters control OF thalamo-striatal pathways implicated in impulse control. As a result of the prefrontal dysfunction, inhibitory response-control functions are impaired and aberrant enhancements in the limbicstriatal motivation system appear, so that patients with $\mathrm{CM}$ and medication overuse behave in a similar way as patients with different addictions, using medication even when it no longer produces rewarding effect (relief of pain) [30]. In contrast, DL dysfunction does not seem distinctive of overusers, being present in all types of migraineurs. Only the ability to arrange by categories, a subtest of the WCST, distinguished negative outcome patients, but this task can also be altered with OF dysfunction [31]. However, normal DL functioning is important in painful conditions, since this region exerts active control on pain perception by modulating corticosubcortical and corticocortical pathways [32]. The role of DL cortex in medication overuse cannot be inferred from our results.

In our study, other factors associated to poor prognosis were the levels of depression, anxiety, and neuroticism traits. Some authors have reported that individual differences in fear and anxiety modulate the pain response and may even cause more suffering [33]. This modulation is exerted by prefrontal mesial and orbitofrontal systems. Impulsivity trait represents a comorbidity that contributes to substance abuse although this specific trait was not overrepresented in our CM group.

This study has some limitations. First, patients with CM were older than EM patients, as reported in epidemiological studies, and age could represent a confounding factor. We performed a lineal model adjusting by age, and found no differences in our results at one-year follow-up. Second, preventive medication varied among the patients in the $\mathrm{CM}$ group. While 11 patients with chronic migraine were not taking any preventive medication because all drugs had failed, other patients were taking two or more (Table 1). We do not believe, however, that preventive medication played a role in their performance on the neuropsychological evaluation. Third, the levels of anxiety and depression were assessed using a self-administered psychometric tool, the Beck anxiety and depression inventory. A complete psychiatric assessment could have detected more precisely the emotional state of the patients, but was out of the scope of this study.

Based on our results, we conclude that $\mathrm{OF}$ dysfunction can be detected in CM patients with medication overuse with appropriate neuropsychological tests. The impairment in $\mathrm{OF}$ tasks predicts a poor prognosis regarding headache outcome and medication overuse in these patients. They would benefit of a neuropsychological examination with emphasis on detecting OF dysfunction, anxiety and depression so that they could benefit of an early and aggressive multidisciplinary therapeutic intervention, such as psychological and psychiatric attention, monthly neurological interviews for treatment follow-up or hospital admissions for analgesic cessation.

Acknowledgments Supported by a grant from the Fondo de Investigaciones Sanitarias, PI 07/0790.

Conflict of interest None of the authors has any conflict of interest regarding this manuscript.

Open Access This article is distributed under the terms of the Creative Commons Attribution License which permits any use, distribution and reproduction in any medium, provided the original author(s) and source are credited.

\section{References}

1. Colas R, Munoz P, Temprano R, Gomez C, Pascual J (2004) Chronic daily headache with analgesic overuse: epidemiology and impact on quality of life. Neurology 62(8):1338-1342

2. Ravishankar K (2008) Medication overuse headache in India. Cephalalgia 28(11):1223-1226

3. Silberstein SD, Olesen J, Bousser MG et al (2005) The International Classification of Headache Disorders, 2nd Edition (ICHDII)-revision of criteria for 8.2 Medication-overuse headache. Cephalalgia 25(6):460-465

4. Halker RB, Hastriter EV, Dodick DW (2011) Chronic daily headache: an evidence-based and systematic approach to a challenging problem. Neurology 76(7 Suppl 2):S37-S43

5. Evers S, Marziniak M (2010) Clinical features, pathophysiology, and treatment of medication-overuse headache. Lancet Neurol 9(4):391-401

6. Fumal A, Laureys S, Di Clemente L et al (2006) Orbitofrontal cortex involvement in chronic analgesic-overuse headache evolving from episodic migraine. Brain 129(2):543-550

7. Hornak J, O'Doherty J, Bramham J et al (2004) Reward-related reversal learning after surgical excisions in orbito-frontal or dorsolateral prefrontal cortex in humans. J Cogn Neurosci 16(3): 463-478

8. Schoenbaum G, Shaham Y (2008) The role of orbitofrontal cortex in drug addiction: a review of preclinical studies. Biol Psychiatry 63(3):256-262

9. Mongini F, Keller R, Deregibus A, Barbalonga E, Mongini T (2005) Frontal lobe dysfunction in patients with chronic migraine: a clinical-neuropsychological study. Psychiatry Res 133(1):101-106 
10. Schmitz N, Arkink EB, Mulder M et al (2008) Frontal lobe structure and executive function in migraine patients. Neurosci Lett 440(2):92-96

11. The International Classification of Headache Disorders: 2nd edition (2004) Cephalalgia 24 (Suppl 1):9-160

12. Beer JS, John OP, Scabini D, Knight RT (2006) Orbitofrontal cortex and social behavior: integrating self-monitoring and emotion-cognition interactions. J Cogn Neurosci 18(6):871-879

13. Heberlein AS, Padon AA, Gillihan SJ, Farah MJ, Fellows LK (2008) Ventromedial frontal lobe plays a critical role in facial emotion recognition. J Cogn Neurosci 20(4):721-733

14. Martin-Rodriguez JF, Leon-Carrion J (2010) Theory of mind deficits in patients with acquired brain injury: a quantitative review. Neuropsychologia 48(5):1181-1191

15. Rankin KP, Gorno-Tempini ML, Allison SC et al (2006) Structural anatomy of empathy in neurodegenerative disease. Brain 129(11):2945-2956

16. Shamay-Tsoory SG, Tomer R, Berger BD, Goldsher D, AharonPeretz J (2005) Impaired "affective theory of mind" is associated with right ventromedial prefrontal damage. Cogn Behav Neurol 18(1):55-67

17. Stone VE, Baron-Cohen S, Knight RT (1998) Frontal lobe contributions to theory of mind. J Cogn Neurosci 10(5):640-656

18. Tombaugh TN (2004) Trail Making Test A and B: normative data stratified by age and education. Arch Clin Neuropsychol 19(2):203-214

19. Zuckerman M, Kuhlman M, Joireman J, Teta P, Kraft M (1993) A comparison of three structural models for personality: the big three, the big five, and the alternative five. J Pers Soc Psychol 65:757-768

20. Beck AT, Steer RA, Ball R, Ranieri W (1996) Comparison of beck depression inventories-IA and -II in psychiatric outpatients. J Pers Assess 67(3):588-597

21. Chiapparini L, Grazzi L, Ferraro S et al (2009) Functional-MRI evaluation of pain processing in chronic migraine with medication overuse. Neurol Sci 30(Suppl 1):S71-S74
22. Stam AH, de Vries B, Janssens AC et al (2010) Shared genetic factors in migraine and depression: evidence from a genetic isolate. Neurology 74(4):288-294

23. Sances G, Galli F, Anastasi S et al (2010) Medication-overuse headache and personality: a controlled study by means of the MMPI-2. Headache 50(2):198-209

24. Lipton RB (2009) Tracing transformation: chronic migraine classification, progression, and epidemiology. Neurology $72(5$ Suppl):S3-S7

25. Valfre W, Rainero I, Bergui M, Pinessi L (2008) Voxel-based morphometry reveals gray matter abnormalities in migraine. Headache 48(1):109-117

26. Scher AI, Stewart WF, Ricci JA, Lipton RB (2003) Factors associated with the onset and remission of chronic daily headache in a population-based study. Pain 106(1-2):81-89

27. Katsarava Z, Schneeweiss S, Kurth T et al (2004) Incidence and predictors for chronicity of headache in patients with episodic migraine. Neurology 62(5):788-790

28. Di Lorenzo C, Di Lorenzo G, Sances G et al (2009) Drug consumption in medication overuse headache is influenced by brainderived neurotrophic factor Val66Met polymorphism. J Headache Pain 10(5):349-355

29. Di Lorenzo C, Sances G, Di Lorenzo G et al (2007) The wolframin His611Arg polymorphism influences medication overuse headache. Neurosci Lett 424(3):179-184

30. Kauer JA, Malenka RC (2007) Synaptic plasticity and addiction. Nat Rev Neurosci 8(11):844-858

31. Freedman M, Black S, Ebert P, Binns M (1998) Orbitofrontal function, object alternation and perseveration. Cereb Cortex $8(1): 18-27$

32. Lorenz J, Minoshima S, Casey KL (2003) Keeping pain out of mind: the role of the dorsolateral prefrontal cortex in pain modulation. Brain 126:1079-1091

33. Ochsner KN, Ludlow DH, Knierim K et al (2006) Neural correlates of individual differences in pain-related fear and anxiety. Pain 120(1-2):69-77 